\title{
THE IDENTIFICATION OF T TAURI-LIKE STARS BY MULTICOLOUR PHOTOMETRY
}

\author{
K. NANDY \\ Royal Observatory, Edinburgh, Scotland
}

\begin{abstract}
A method of distinguishing between T Tauri-like stars and reddened early type field stars by the application of $U B V R I$ photometry is described, and the results for T Tauri-like variables in NGC 2264 are presented. The plates have been measured using the new measuring machine GALAXY. $(V-R)$ colours, which are less affected by spectral peculiarities, are used to determine infrared and ultraviolet excesses. The range of variability in $U B V R I$ colours of these varables has also been studied.
\end{abstract}

\section{Introduction}

The young clusters and associations contain a large number of $\mathrm{T}$ Tauri-like variables which populate the elevated main sequence. These stars are known to belong to the contracting phase of the main sequence. It has become evident from the work of Mendoza $(1966,1968)$ and Low et al. (1970) that these stars are intrinsically brighter in the infrared and ultraviolet than expected from their spectral types. Because of their intrinsic spectral peculiarities, the separation of these stars from reddened field stars presents a problem. In this paper, a method distinguishing between $\mathrm{T}$ Tauri-like stars and reddened early type field stars by the application of $U B V R I$ photometry is described, and the results for T Tauri-like variables in NGC 2264 as regards their infrared and ultraviolet excesses, and the range of variability in $U B V R I$ colours are presented.

\section{Observations and Reductions}

The observational material was obtained with the $40 / 60 / 150 \mathrm{~cm}$ Schmidt telescope of the Royal Observatory, Edinburgh's outstation at Monte Porzio during the period from March 1968 to February 1969. For $U, B, V, R$ and $I$ magnitudes the following emulsion filter combinations are used:

$$
\begin{array}{ll}
U & \text { IIaO + UG2 (2 mm Schott) } \\
B & \text { IIaO+GG13 (2 mm Schott) } \\
V & \text { IIaD+GG14 (2 mm Schott) } \\
R & \text { IaE + Ilford colour filter 204 } \\
I & \text { IN + Ilford colour filter 207 }
\end{array}
$$

$U, B$ and $V$ plates are calibrated by photoelectric sequences of Walker (1956). For $R$ and $I$ magnitudes photographic calibration has been achieved by using a pair of doubly refracting calcite plates in conjunction with a polaroid (Brück et al., 1969). (For future work photoelectric $R$ and $I$ magnitudes are now being obtained with the Observatory's Twin telescope). The plates have been measured using the Observatory's new measuring machine GALAXY. The calibration curve obtained from the GALAXY 


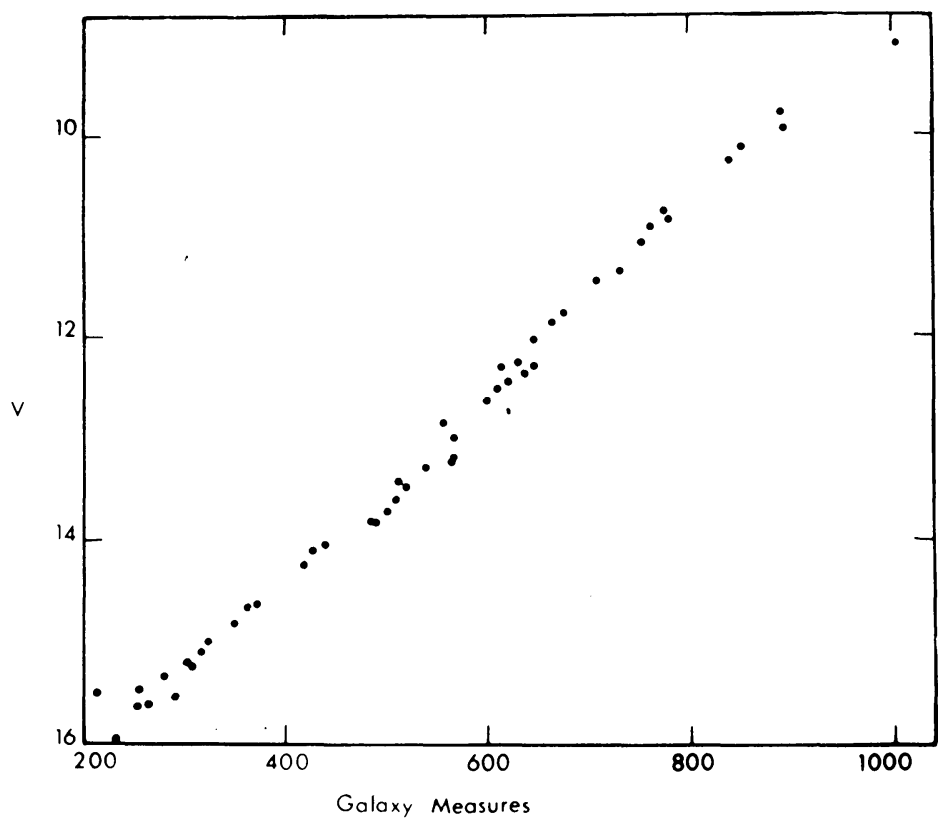

Fig. 1. The calibration curve obtained from the GALAXY measures.

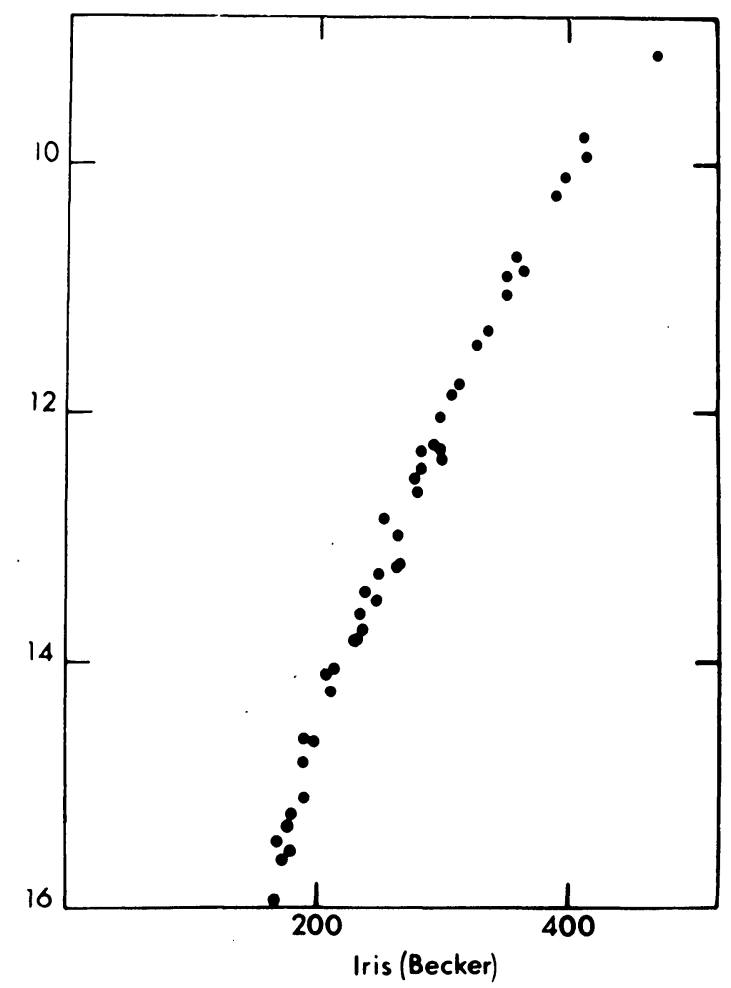

Fig. 2. The calibration curve derived from the iris photometer readings. 
measures is shown in Figure 1 (For comparison, Figure 2 shows the calibration curve derived from the conventional iris photometer readings, the same plate being used). It is to be noted (cf. Figure 1) that the GALAXY measures are linear with magnitudes $(-2.5 \log I)$ over the magnitude range from $V=8^{\mathrm{m}} \cdot 0$ to the plate limit.

\section{Identification of T Tauri-like Stars}

The separation of T Tauri-like stars from reddened field stars can be achieved in the following way: if the stars are intrinsically brighter in the ultraviolet and infrared than normal for their spectral type as indicated by their absorption lines, they occupy the same region of the $(U-B, B-V)$ diagram as highly reddened early type stars. However, the $(U-V),(V-R)$ and $R-I)$ colours can be predicted for the early type stars from the parameter $Q$ defined as $Q=(U-B)-0.70(B-V)(Q$ determines the spectral type and the extinction law being known (Nandy, 1968) the colour excesses $E_{B-V}, E_{V-R}$, and $E_{V-I}$ can be computed). In the case of reddened early type stars the predicted colours would agree with the observed $(V-R)$ and $(V-I)$ colours. On the other hand, if the difference between the predicted and observed colours is much larger than the errors of observations, the stars are likely to be of later types with anomalous colours characteristics of $\mathrm{T}$ Tauri stars. For these stars $(V-R)$ colours corrected for interstellar reddening, which are less affected by peculiarities in the stellar spectra, are found necessary to estimate their spectral types, and infrared and ultraviolet excesses can then be directly computed. The spectral types of $\mathrm{T}$ Tauri-like stars determined from $U B V R I$ photometry and from low dispersion objective prism spectra as faint as $V=12^{\mathrm{m}} \cdot 5$ agree within $\frac{1}{2}$ spectral class.

\section{Sources of Error and Range of Variability}

The main sources of error is the variability of the stars, since $U, B, V, R$ and $I$ magnitudes have not been obtained simultaneously. During the period of observations a considerable number of plates in each colour have been obtained. The range of variability (in amplitude) of T Tauri-like stars in each colour is estimated by the intrinsic rms dispersion of the mean magnitudes. It is found that the rms dispersion in $U$ magnitudes of the $\mathrm{T}$ Tauri-like variables is much larger than that in $I$ magnitudes (Nandy and Pratt, 1972). The mean rms dispersion in $U B V R I$ colours obtained for the known T Tauri-like stars in the cluster NGC 2264 is as follows:

\begin{tabular}{|c|c|c|c|c|c|}
\hline & $\bar{\sigma}_{U}$ & $\bar{\sigma}_{B}$ & $\bar{\sigma}_{V}$ & $\bar{\sigma}_{R}$ & $\bar{\sigma}_{I}$ \\
\hline $\begin{array}{l}\text { T Tauri-like stars } \\
\text { Photometric error as deter- } \\
\text { mined from rms. error of } \\
\text { standard stars }\end{array}$ & $\begin{array}{l}0.6 \pm 0.4 \\
\pm 0.08\end{array}$ & $\begin{array}{l}0.4 \pm 0.3 \\
\pm 0.06\end{array}$ & $\begin{array}{c}0.20 .1 \\
00.04\end{array}$ & $\begin{array}{c}0.15+0.1 \\
\therefore 0.06\end{array}$ & $\begin{array}{c}0.1+0.1 \\
+0.07\end{array}$ \\
\hline
\end{tabular}


Only those plates which were taken in a small interval of time ( 1 week) have been used to determine $(V-R)$ colour, and therefore the standard error of mean $(V-R)$ colour is not more than \pm 0 . 2 .

\section{Ultraviolet and Infrared Excesses}

The ultraviolet, blue and infrared excesses are defined as follows:

$$
\begin{aligned}
\delta(U) & =(U-V)_{\mathrm{obs}}-(U-V)_{\mathrm{predicted}}-E_{U-V} \\
\delta(B) & =(B-V)_{\mathrm{obs}}-(B-V)_{\mathrm{predicted}}-E_{B-V} \\
\delta(I) & =(V-I)_{\mathrm{predicted}}-(V-I)_{\mathrm{obs}}+E_{V-I} .
\end{aligned}
$$

The variables, in particular, the $\mathrm{H} \alpha$ emission stars in NGC 2264 exhibit infrared and ultraviolet excesses and there appears to be a positive correlation between them (Nandy, 1970). These stars, as Mendoza (1968) pointed out, are more luminous than indicated by their colour magnitude diagram. Their mean infrared excesses are comparable to the T Tauri-like stars with $L_{\text {total }} / L_{\mathrm{sp}} \sim 3$. Masses and ages of these variables computed on this assumption (Nandy, 1970) are shown in Figure 3. It is found that their masses range from one to two solar masses, and the average age is $\sim 10^{6} \mathrm{yr}$, in agreement with the estimated age of the B stars of the cluster.

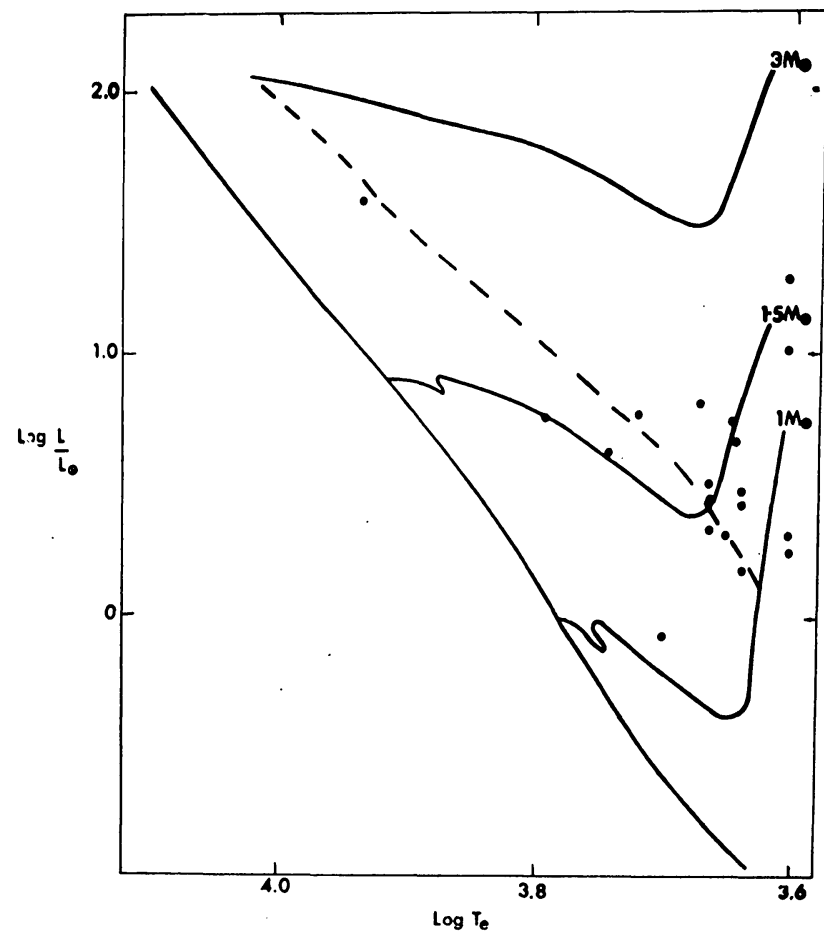

Fig. 3. H-R diagram for T Tauri-like stars in NGC 2264 as studied here. The dashed line indicates the locus of stars $10^{6} \mathrm{yr}$ old. 


\section{Dust Model for T Tauri-Like Stars}

The indication that the range in variability in $I$ is much less than that in $U$, and the infrared excesses and ultraviolet excesses are correlated, favour the model of circumstellar dust clouds for T Tauri-like stars (Low et al., 1966) in which grains absorb the visual radiation and radiate in the infrared.

The extinction law for the circumstellar dust cloud around $\mathrm{T}$ Tauri-like stars may be very much different from those for the general interstellar medium, since the physical conditions in the dense cloud favour the growth of large particles. Hartmann (1970) has suggested that planetisimal particles (iron grains coated with silicates) may be formed in these dense clouds. The theoretical extinction curves for such particles have been computed for various sizes of mantles (Nandy and Wickramasinghe) and it is found that for large sizes of mantles the extinction in ultraviolet becomes nearly non-selective (Figure 4). The simultaneous existence of infrared and ultraviolet excesses may be explained by the fact that large planetisimal particles are relatively more transparent in the ultraviolet (c.f. Figure 4) and radiate more efficiently in the infrared. However, until we get more observational data as regards ultraviolet and infrared excesses of $\mathrm{T}$ Tauri-like stars in cluster and associations, these theoretical results remain tentative.

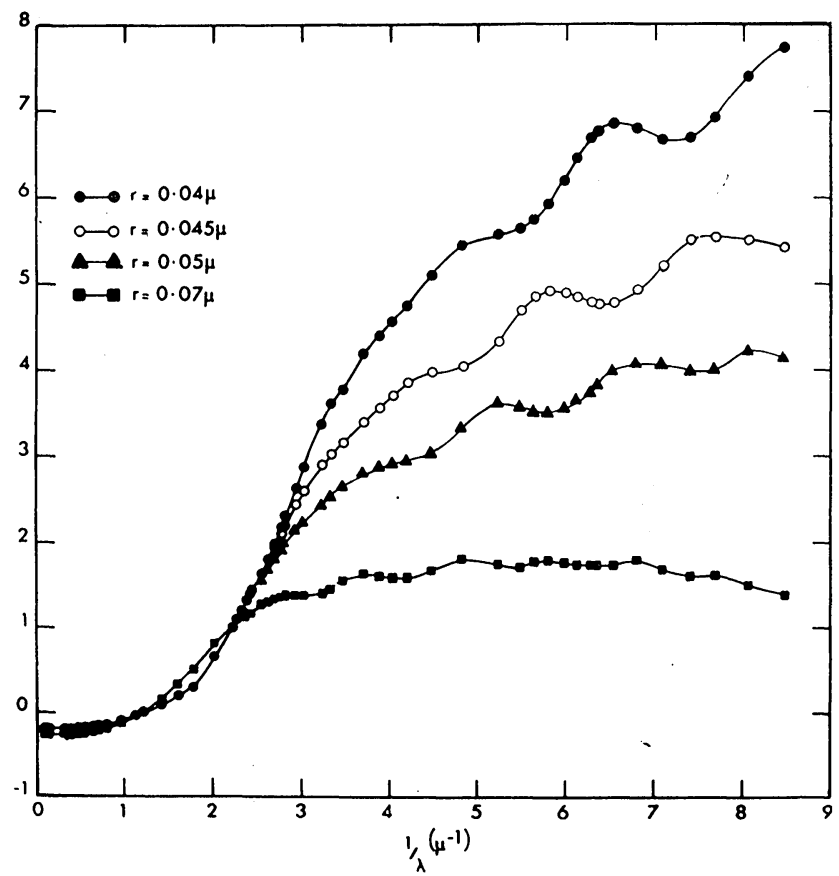

Fig. 4. Theoretical extinction curves for planetisimal particles (iron grains coated with silicates). 


\section{References}

Brück, M. T., Caprioli, G., Nandy, K., and Smiglio, F.: 1969, Astrophys. Space Sci. 4, 213.

Hartman, K. W.: 1970, 'Évolution stellaire avant la séquence principale', Soc. Roy. Sci. Liège, Se série 19, Institut d'Astrophysique, Cointe-Ougrée, Belgique.

Low, F. J., Johnston, H. L., Kleinman, D. E., Latham, A. S., and Geisal, S. L. : 1970, Astrophys. J. $160,531$.

Low, F. J. and Smith, B. J!: 1966, Nature 212, 675.

Mendoza, E. E.: 1966, Astrophys. J. 143, 1010.

Mendoza, E. E.: 1968, Astrophys. J. 151, 977.

Nandy, K.: 1968, Publ. Roy. Obs. Edinburgh 6, 169.

Nandy, K.: 1971, Publ. Roy. Obs. Edinburgh 7, 47.

Nandy, K. and Pratt, N. M.: 1972, Astrophys. Space Sci. 19, 219.

Nandy, K. and Wickramasinghe, N. C.: 1973, Astrophys. Space Sci., in press.

Walker, M. F.: 1956, Astrophys. J. Suppl. 2, 365. 